\title{
Politique
}

Politique

\section{S. Amin, G. Arrighi, A.G. Frank, I. Wallerstein, La crise, quelle crise?, Paris, Maspéro, 1982, 244 p.}

\section{Denis Perreault}

Numéro 6, automne 1984

La réforme des institutions politiques

URI : https://id.erudit.org/iderudit/040473ar

DOI : https://doi.org/10.7202/040473ar

Aller au sommaire du numéro

\section{Éditeur(s)}

Société québécoise de science politique

ISSN

0711-608X (imprimé)

1918-6584 (numérique)

Découvrir la revue

Citer ce compte rendu

Perreault, D. (1984). Compte rendu de [S. Amin, G. Arrighi, A.G. Frank, I.

Wallerstein, La crise, quelle crise?, Paris, Maspéro, 1982, 244 p.] Politique, (6),

200-204. https://doi.org/10.7202/040473ar d'utilisation que vous pouvez consulter en ligne.

https://apropos.erudit.org/fr/usagers/politique-dutilisation/ 
S. Amin, G. Arrighi, A.G. Frank, I. Wallerstein, La crise, quelle crise?, Paris, Maspéro, 1982, 244 p.

Le titre de cet ouvrage reprend l'interrogation sur un thème on ne peut plus vidé de son contenu analytique. C'est pour contrer ce "galvaudage» discursif que ces quatre théoriciens économistes 
partagent ce débat. Leur but: promouvoir un dépassement de cette (ou ces) crise(s) vers la démocratie et l'égalité. Pour ce faire, ils dénoncent un éventuel redéployement de l'accumulation inlassable et ininterrompue du capital.

Amin, Arrighi, Frank et Wallerstein nous proposent un questionnement à plusieurs niveaux: la crise comme stagnation économique? la crise comme crise structurelle? crise d'hégémonie? crise de transition? crise des rapports Nord/Sud? crise de l'impérialisme?

Immanuel Wallerstein jette, dans le premier texte, quelques prémisses en ce sens. L'auteur analyse la période actuelle comme une période de récession qu'il qualifie de stagnation économique "que d'aucuns appellent aussi «la crise». Il faut bien voir qu'une telle stagnation n'a rien de nouveau (puisque le mouvement des oscillations économiques est cyclique depuis plusieurs siècles) et que la stagnation actuelle n'est pas la crise, bien qu'elle l'accentue et la rende plus évidente à tous" (10).

Pour Wallerstein, ce qu'il faut retenir sur la période actuelle, qu'il qualifie de transition, est le fait "que le monde se trouve au milieu d'une crise structurelle... qui porte sur le long terme» (11). Une crise qui doit être comprise comme le déclin de l'économiemonde capitaliste, système politico-économique qui a émergé il y a un siècle avec le déclin du féodalisme. Ce système repose sur la curieuse logique qui fait de l'accumulation un but en soi. Nous ne traversons à l'heure présente qu'une aggravation des conditions du système en crise.

Pour étoffer son argumentation, Wallerstein soulève quelques hypothèses. D'un côté, il considère que ce ne sont pas les échecs de notre système historique qui ont entraîné sa crise mais ses succès. "Après avoir fonctionné et prospéré pendant cinq siècles, ce système est maintenant en train de se désintégrer, au moment même où il atteint un niveau d'efficacité sans précédent»(12). 
Et de l'autre, que les contradictions fondamentales du système résident dans le déséquilibre global de l'offre et de la demande. Tôt ou tard, l'efficacité du système élève l'offre de biens à un niveau où la demande devient insuffisante «et l'économie-monde capitaliste connaît l'un de ses traditionnels goulots d'étranglement de l'accumulation» (16).

Le dépassement oblige une restructuration basée sur des solutions d'ajustement. Les deux principaux mécanismes d'ajustement identifiés par l'auteur - la prolétarisation et l'expansion géographique - possèdent leurs propres limites. Comme il l'indique, quelle que soit l'efficacité de ces mécanismes, ils ont trop facilement supposé une offre illimitée de main-d'œuvre qui n'existe pas en réalité.

En conclusion, Wallerstein précise que l'élément décisif de cette transition vers un nouvel ordre mondial inscrite dans les limites du capitalisme, est la montée des mouvements antisystémiques où Octobre 1917 fut le saut qualitatif. Une conclusion qui nuance quelque peu l'impression laissée par les différentes propositions de son analyse, à savoir le volontarisme de l'histoire, le sens inéluctable vers l'homme nouveau. «La crise est objective et poursuit son cours. Mais sa résolution dépend de notre intervention humaine, collective, et n'est pas déterminée» (56).

Pour sa part, Giovanni Arrighi nous soumet une définition de la crise, crise d'hégémonie qui se démarque de la précédente en ce qui a trait aux caractéristiques de la stagnation économique présente, vue surtout comme une nouvelle période de changement discontinu. La crise actuelle est une phase, un stade de transition d'une économie-monde capitaliste vers un nouvel ordre mondial. Arrighi parle de crise longue qui n'exclut pas pour autant la possiblité de crises courtes appelées changement discontinu, qui dans la mesure où elles sont de plus en plus longues et fréquentes peuvent être qualifiées de crise de transition. 
Pourquoi parler de crise de transition? L'auteur nous répond: «Le changement le plus spectaculaire et le plus brutal des douzes dernières années a été la crise de l'ordre politico-économique mondial instauré après la seconde Guerre Mondiale sous l'hégémonie des États-Unis» (59). Une hégémonie qui a comme but primordial «...moins de garantir la sécurité des échanges que celle de l'entreprise capitaliste, notamment face aux menaces de nationalisation» (61).

Par un détour touchant l'expansion transnationale du capital, l'accroissement du pouvoir de négociation de la main-d'œuvre sur les lieux de travail, l'auteur pose la constatation suivante: nous assistons surtout à une crise de sécurité d'expansion du capital plutôt qu'à une crise d'autorité-mondiale. Cette constatation l'amène à présenter des scénarios pour les années 80 et à soulever la difficulté de prédire le sens de cette crise d'hégémonie. Peut-elle déboucher sur un nouvel ordre mondial? "Ce qui est sûr, en tout cas, c'est que, jusqu'à présent, aucune alternative politique viable... n'a réussi à s'imposer dans la politique mondiale» (109).

André Gunder Frank quant à lui, indique que la crise si profonde soit-elle ne signifie pas la fin du système. "Au contraire, la "crise» désigne cette période durant laquelle de nouvelles possibilités d'adaptation doivent permettre d'éviter cette fin, qui n'intervient que lorsque toutes ces possibilités ont été épuisées» (110). Il propose ici la possibilité pour le système de créer de nouvelles adaptations qui lui assurent à l'infini de nouveaux cycles de vie. Il tompt, si l'on peut dire, avec l'analyse de Wallerstein sur l'inévitabilité objective de la destruction, de la dégénérescence du système.

Tout en acquiesçant, dans l'ensemble, aux différentes caractéristiques définissant et expliquant la crise des trois autres auteurs, Frank reste sceptique face au potentiel révolutionnaire des forces antisystémiques. C'est le caractère antisystémique qu'il remet en cause ici. Les alternatives quoique importantes, entre autres éco- 
logistes, féministes, en se renforçant s'adaptent surtout aux exigences du capital.

Dans le dernier texte, Samir Amin nous ramène à la dimension historique privilégiée par les deux premiers auteurs. Il propose, après avoir divisé l'histoire du capitalisme en trois phases, que 1880 est la dernière rupture. Cette rupture installe le système impérialiste. La crise actuelle est la coupure d'avec ce système, la crise de l'impérialisme! «La crise contemporaine s'inscrit dans la longue transition du capitalisme au socialisme. Ce n'est pas seulement une crise économique des pays capitalistes avancés, ni même seulement une crise de la DIT Nord/Sud. C'est aussi la crise des deux hégémonismes: celui des États-Unis sur le déclin (même si le déclin est théoriquement réversible) et celui de l'URSS, aux prises avec l'aspiration des peuples de l'Europe de l'Est...» (228).

Amin voit en cette double crise une nouvelle étape pouvant favoriser une longue période de révoltes populistes et de désintégrations qui, loin de permettre une relance du capitalisme, ouvre au contraire des perspectives socialistes. Amin à l'opposé de Frank et malgré quelques démarcations importantes, souscrit à la marche de l'homme vers le socialisme.

Outre le caractère globalisant et souvent simplificateur des propos (qui somme toute sont nécessaires pour rendre plus limpide une réalité des plus complexe), on peut trop aisément reprocher à cet ouvrage de n'apporter que très peu d'éléments nouveaux au débat, surtout pour ceux qui connaissent les différentes thèses de ces auteurs largement diffusées à travers leurs publications antérieures. Ce livre a tout de même l'immense avantage de réunir en un seul ouvrage des thèses qui par leur somme portent l'analyse à un niveau de transparence essentielle pour construire une alternative. La crise, quelle crise?, un instrument stimulant à conserver.

Denis Perreault Chercheur, GRAPP 